\title{
Patient perspectives on the treatment for Hashimoto's thyroiditis: a qualitative analysis
}

\author{
Stephanie A Lee ${ }^{1 *}$, Nichole E Stetten ${ }^{1}$ and Stephen D Anton ${ }^{2}$ \\ ${ }^{1}$ Department of Social and Behavioral Sciences, College of Public Health and Health Professions, University of Florida, Gainesville, USA \\ ${ }^{2}$ Department of Aging and Geriatric Research, College of Medicine; Department of Clinical and Health Psychology, University of Florida, Gainesville, USA
}

\begin{abstract}
Hashimoto's thyroiditis (HT) is an autoimmune disorder which can be accompanied by a myriad of symptoms. Conventional medicine currently treats only one of these symptoms, hypothyroidism, with thyroid hormone replacement therapy. The focus of this project was to obtain personal views from HT patients on their specific health care plan, satisfaction of the treatment offered, and overall burden of this disease. Individual semi-structured interviews were conducted to obtain this information. Emerging themes included patient experiences with health care providers, symptoms experienced by patients, and treatment options offered to patients. Results suggest patient-centered care is absent from their experiences in various health care settings and current protocol for management of HT is not sufficient for alleviating many disease-related symptoms. Participants often look beyond their health care professional to better understand the disease and to seek out additional treatments. Complementary treatment options to standard therapy were strongly desired. Obtaining direct insight from the patient's perspective could be valuable for strengthening patient-provider relationships. Health care providers being mindful, informative, and empathic of patient views could improve patient's quality of care and encourage further treatment possibilities for individuals who suffer from symptoms of Hashimoto's.
\end{abstract}

Abbreviations: HT: Hashimoto's Thyroiditis; TSH: Thyroid Stimulating Hormone; MDM: Minimally Disruptive Medicine

\section{Introduction}

Hashimoto's thyroiditis (HT), also known as chronic lymphocytic thyroiditis or autoimmune thyroiditis, is now considered the most prevalent autoimmune disease and endocrine disorder [1]. One of the symptoms in individuals with HT is hypothyroidism, a deficiency of thyroid hormones that disrupts such things as heart rate, body temperature, and metabolism. Current medical protocol for treatment of HT is supplementation with the synthetic thyroid hormone, Levothyroxine [2]. This medication is only warranted if the thyroid stimulating hormone (TSH) is out of range. Symptomatic distress is often still reported independent of thyroid dysfunction $[3,4]$ including weight gain, hair loss, cold intolerance, impaired cognitive function, and extreme fatigue.

Although conventional medicine does not offer standard treatment of HT symptoms outside of hypothyroidism, various complementary health approaches such as acupuncture [5], diet modification [6], and trace element supplementation [7] do offer treatment options in efforts to suppress the autoimmune response.

The purpose of this pilot study is to uncover the perceived level of HT related symptom load in euthyroid patients and treatment plans offered to alleviate symptoms identified by either their physician or through self-treatment. The patient's interpretation and satisfaction level of the relationship with their past and present health care provider(s) treating them for HT will also be explored.

\section{Methods}

This study used semi-structured interviews (Table 1) with individuals diagnosed and treated for HT by a health care provider.
The definition of health care providers in this study refers to any health specialist who provides preventative or curative health care services to individuals. The purpose of these semi-structured interviews was to expose the patient's perception of the level of satisfaction from various health care providers they have received regarding treatment for symptoms associated with HT and how it has impacted their health related quality of life.

\section{Theoretical Model}

The Minimally Disruptive Medicine (MDM) model was used as a theoretical guide when developing interview questions period (Figure 1). The model's concepts aim to understand and advance patient goals for health, health care, and life in a personalized manner by presenting as an illustration of care for and about the whole person on an individual basis. The model's concepts are operationalized through specific interventions aimed at patients, clinicians, care teams, among others [8] because these all must work together to reach the goal of improved health related quality of life and symptom relief for the patient. Interview questions were modeled to cover these concepts. The MDM model is a patient-centered approach based on providing the "smallest possible treatment burden on patients" [8] while offering effective treatment and service provisions [9]. This model was also used to guide the thematic analyses.

${ }^{\star}$ Correspondence to: Stephanie A. Lee, Department of Social and Behavioral Sciences, College of Public Health and Health Professions, University of Florida Gainesville, FL 32601, USA, Tel: (352)-273-6214; E-mail: Stephanie.lee@phhp.ufl.edu

Key words: autoimmune, Hashimoto's, hypothyroid, patient centered care, thyroiditis

Received: June 18, 2018; Accepted: June 28, 2018; Published: June 30, 2018 


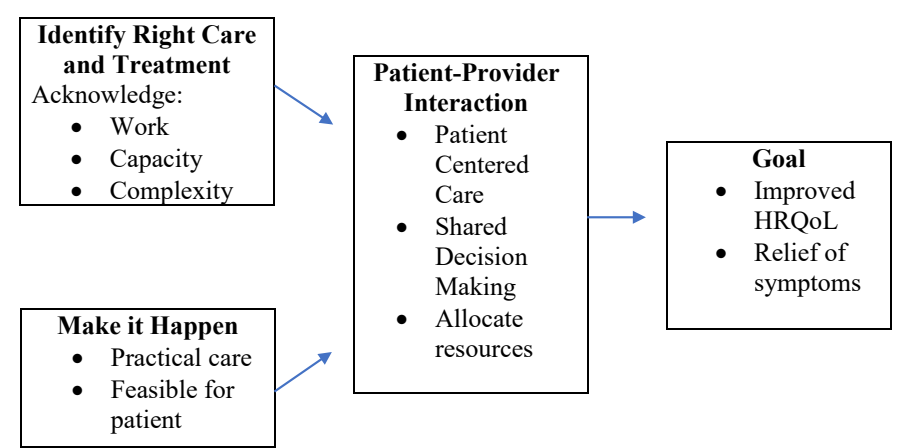

Figure 1. Concept model of the categories and themes from interviews using the Minimally Disruptive Medicine model

Table 1. Initial topic guide for semi-structured patient interviews

- What kind of health care provider do you currently see?

- How many providers have you seen for your symptoms? What kinds?

- How long did it take to get your Hashimoto's diagnosis?

- What kind of provider gave you the diagnosis? How long ago?

- Have any providers explained the symptoms or prognosis for Hashimoto's?

- What symptoms have you told your provider about?

- Do you feel your provider listens when you report symptoms?

- Are you satisfied with the treatment options offered to you? What are they?

- Have your symptoms subsided with this treatment(s)?

- How satisfied are you with your current provider?

- Have you sought self-treatment? If so, where did you get your information?

- Has your self-treatment helped with your symptoms?

- Explain the self-treatment.

- Have you discussed the self-treatment with your provider? What was the reaction?

- How has your quality of life changed since your diagnosis?

\section{Participants}

This study was approved by the Institutional Review Board at the University of Florida. Recruitment was initiated by disseminating a flyer advertising the study at the university hospital and throughout the University of Florida's campus. The flyer was also circulated electronically on Facebook, to reach a broad participant pool. Through the use of social media, four different states were represented in the sample.

Participants were screened through email was used to verify study eligibility. Inclusion criteria included individuals age between 18-45, clinically diagnosed with HT, and currently being treated by a health care provider. Participants were excluded if they had co-occurring autoimmune diseases, currently pregnant, on birth control, or a heavy drinker ( $>3$ drinks per day or $>15$ per week). Six total individual interviews were conducted and ranged from 15 to 47 minutes. All interviews were held over the phone, and recorded using the "Tapea-Call" mobile application. Participant characteristics are shown in Table 2. Interviews were downloaded, transcribed verbatim, and deidentified.

\section{Analysis}

The constant comparison method was used to analyze the data and develop a grounded theory [10]. Grounded theory analysis begins with open coding, which is defined as "the process of breaking down, examining, comparing, conceptualizing, and categorizing data" [10,11]. Open coding and additional sampling occurs until saturation (no new data) is met $[10,11]$. A trained researcher (SL), open coded all of the interviews and discovered major themes. The central themes were then mapped within the constructs of the MDM model. Analysis was then reviewed by a qualitative consortium. Open coding was completed by hand instead of using data mining software, in order to take on the full context of the interviews.

\section{Results}

Through the constant comparison method, three themes emerged:

1) patient experiences with health care providers, 2) symptoms experienced by patients, and 3) treatment options offered to patients. Below we describe the key findings related to each of these themes.

\section{Patient Experiences with Health Care Providers}

Interviews revealed the term 'health care provider' included conventional medicine (primary care physicians and referred specialists), alternative medicine, and integrative medicine. HT patients saw a plethora of health care providers before obtaining a diagnosis and then continued on to numerous specialists to treat individual symptoms. An average of five providers were seen before diagnosis of HT was even given. Primary care physicians often referred out to endocrinology, who are known to be the first line of defense in HT treatment in conventional medicine.

When asked what type of physician gave them the HT diagnosis, answers were given such as "my primary care doctor" or "my gynecologist told me I might have it, so I asked my regular doctor to test me." Another participant said "I knew something was wrong with me, and they tried telling me I had depression and sent me to a psych doctor who said I should have my thyroid checked."

After the HT diagnosis all six of the participants were referred to an endocrinologist, although only two continue to see them for routine follow-ups. One participant said "I knew more about my disease than that endo doctor. I had to find someone else that looked at more than a lab test. I found an integrative medicine doctor after that, and it's much better."

There was a high frequency of health care provider turnover rate in the HT patient population interviewed. We found this was not exclusive to specialist referrals, as most participants switched providers within departments at various points of treatment.

Once diagnosed, HT patients were generally referred to endocrinologists who specialize in thyroid disorders even though rheumatologists focus on autoimmune diseases. Rheumatologists refused to treat four of the participants. The goal of HT treatment

Table 2. Patient demographics

\begin{tabular}{|l|l|}
\hline Characteristics & Frequency, n (\%) \\
\hline Gender & \\
\hline Female & $5(83)$ \\
\hline Male & $1(17)$ \\
\hline Location & $2(33)$ \\
\hline Rural & $4(67)$ \\
\hline Urban & \\
\hline Age in Years & $0(0)$ \\
\hline $18-25$ & $1(17)$ \\
\hline $26-30$ & $1(17)$ \\
\hline $31-35$ & $3(50)$ \\
\hline $36-40$ & $1(17)$ \\
\hline $41-45$ & \\
\hline Years with Hashimoto's & $1(17)$ \\
\hline $0-5$ & $4(67)$ \\
\hline $6-10$ & $0(0)$ \\
\hline $11-15$ & $1(17)$ \\
\hline $16-20$ & $0(0)$ \\
\hline$>20$ & \\
\hline
\end{tabular}


in endocrinology is to stabilize the TSH by consistently adjusting thyroid hormone medication until optimal. However, patients were not being treated for any other symptoms other than hypothyroidism. A participant stated "I was just a number to my endocrinologist. She didn't care about how I was feeling or what symptoms I was having; she only looked at the numbers." This person chose to get a second opinion in endocrinology, however had the same outcome and was left feeling frustrated. This was the general consensus in all six interviews concerning endocrinology.

Patients who were unsatisfied with their endocrinologist felt stressed from dealing with a chronic illness seemingly alone. Feeling unheard and insignificant when explaining their symptoms from HT made the situation worse. "She made me feel like I was going crazy; she told me my hair loss and fatigue had nothing to do with my disease because I was taking Levothyroxine. I just wanted to see another doctor that would listen." Another participant stated, "I pay out of pocket to see doctor after doctor hoping one will treat me like a person and see that I am not ok." Provider satisfaction rates were verbalized as low in this category, and there was a lack of perceived patient centered care.

Integrative medicine providers had the most positive responses as far as patient satisfaction and long-term care. "I cried at my first visit with my integrative physician. She treats the patient, and not the disease." This sentiment was shared by other participants with statements regarding integrative physicians such as "we talked about other things besides thyroid pills" and "after seeing three endocrinologists I will never go again, all people with this disease should just go to this kind of doctor (integrative physician). They get it." Response to patient concerns and listening to individualized symptoms had a profound effect on the morale of the participants. The patientprovider interaction was positive in these cases and, in turn, relieved the patient of frustration and gave them hope for relief of symptoms.

\section{Symptoms Experienced by Patients}

The study aimed to find what symptoms were experienced in participants and if any had subsided now that their thyroid was at an acceptable functioning state (euthyroid). The definition of symptoms in this text refer to any physical or mental subjective evidence that warrants complaint, discomfort, or change in everyday life.

The interviews revealed that for most patients, before diagnosis the first complaint to a health care provider was unexplained fatigue, followed closely by weight gain. When asked about initial symptoms, a participant stated "I thought I was either pregnant or dying from cancer. I never felt so tired in my life." Another woman stated "I feel like I'm not the same person I was before this disease; I keep gaining weight and am uncomfortable in my own skin." These symptoms affected the sleeping pattern of the participants, their family responsibilities, work schedule, and sense of self.

Wondering if any symptoms were alleviated after a stable dose of thyroid hormone replacement was found, we received the following responses: "no, I felt like death every day and the doctor told me my thyroid was working fine and it's all in my head." "I didn't even notice a difference when my thyroid got so-called normal. I am nearly bald and my husband thinks I am getting dementia because I can't think straight half the time (due to brain fog)." For one participant, she did say stabilizing her thyroid hormones did help. "I noticed my naps were getting shorter and I could read a book and remember it the next day." The interviews revealed symptoms outside of hypothyroidism often continue regardless of hormone replacement therapy.
The interviews had a dominating symptom which every participant talked about, fatigue. It was also the most problematic symptom even after treatment has been established. "I sleep a lot and my family suffers because of that." With HT disproportionately affecting women and having an average onset age of 35, this is hitting mothers who often have young children to care for. "I am too tired to chase after my 7-year-old...I get delirious from fatigue and can't even drive."

Quality of life can be drastically reduced from fatigue and is prevalent in the HT population interviewed. "Fatigue is the worst part of Hashimoto's. I have to nap in my car at my job because I can't make it through the day without one." It has also shown to affect relationships with spouses. "I'm too tired to go to the gym with my husband, to cook him dinner, to have sex with him...it is just too much and all I want to do is sleep." This symptom in particular can change a person's quality of life considerably; comments in the interviews suggest a distinct difference in level of activity and overall enjoyment of life from before onset of HT.

\section{Treatment Options Offered to Patients}

The definition of treatment in this text refers to any behavioral or medical intervention aimed to alleviate symptomology of HT. As many HT patients still complain of symptoms after a euthyroid state is reached from prescription medication, we sought out to see if there were any other unconventional treatments being offered to patients by providers, or if they are self-treating through independent methods.

We asked if any providers had other recommendations for symptoms. One participant said "my doctor told me there is no other treatment and I should be fine." Another commented "no, I was told that my other symptoms had nothing to do with Hashimoto's." A strong response to this interview question from participants was the recommendation from integrative and functional medicine doctors for supplement use. Ordering labs to confirm possible deficiency of such elements as zinc, selenium, Vitamin D and magnesium were done as part of regular HT treatment for these health care providers. "I didn't realize how low my numbers were for those basic vitamins and minerals. I'm glad she (the provider) thought to have those tested."

Self-treatment was a prominent theme in all interviews. All participants have used the internet to learn more about HT and what to expect for this lifelong chronic illness. Other reasons for internet use were to find online support groups and to gain help in understanding how to read lab test results. When asked what self-treatment the participant has tried, if any, we received a strong response for both dietary intervention and altering environmental surroundings. "There is an anti-inflammatory diet that is all over the internet and I think I am going to look into that." Social media also seemed to be a popular entity for HT patients to use for self-guidance. "There's like 50 different support groups on Facebook and all of them offer suggestions on how to feel better. Some of these online groups let you post your actual lab tests and other members help you read them." Lastly, another answered "oh man, let me tell you; there are people recommending acupuncture, essential oils, and even eating organ meat to help with these insane symptoms. To be honest, I'd try them all if it meant I would feel normal again." Comments suggest HT patients are open to new treatments and anxious for a strong social support group of others who suffer from HT.

Although not recommended by conventional medicine practitioners, dietary change for the treatment of HT was common in the interviews. Uncovering why this treatment was considered and where they are gaining information about the relationship between 
diet and HT was obtained. "My doctor offered no help whatsoever so I researched myself online. A lot of people were talking about avoiding gluten and dairy and I have nothing to lose so why not."

There is currently no scientific data regarding dietary influence on symptoms of HT, and this is likely why it is not part of treatment in conventional medicine. "I asked my doctor about diet and they said it doesn't matter what I eat it makes no difference. But it sure does make me feel better when nothing else would." Another commented "A nurse practitioner told me to stop eating gluten and limit my sugar." Participants often reached outside of the medical community to seek treatment and reported success in relieving some symptoms by modifying their diet.

Dietary change is demanding and difficult for most people to adhere to over long periods of time. However, the willingness to at least try this potential treatment was astounding due to the eagerness for participants to feel relief. "I stopped eating everything I love because I read it worked for other people. It makes going out restaurants and parties really hard but having Hashimoto's is harder." A few participants did get recommendations from their integrative medicine providers about changing how they eat. "My integrative doctor gave me a bunch of handouts and told me I have to heal my gut." Another person stated "She said all symptoms are from food and the goal is to reduce inflammation. I would eat dirt if she told me I'd have my life back." Participants were eager and excited by the possibility of treatment outside of hormone replacement, although they did understand medication would still be needed for life. The interviews clearly showed HT patients want more than a one-size-fits-all treatment to this very individualized disease.

\section{Limitations}

The biggest limitation to this study, is that only six interviews were conducted. Although only six individuals were interviewed, saturation (no new themes emerged) was reached among those participants, showing appropriate sampling for a pilot study. A follow up study could include more interviews with larger demographics. This study also contained mostly females; although HT disproportionately affects women, men can also develop the disorder. Only one trained interviewer was used, and additionally completed the coding and analysis. However, the results were presented and reviewed by a qualitative consortium. This consortium consisted of two qualitative content experts and many other researchers experienced in qualitative research. This study served as a pilot for the interview guide.

\section{Conclusion}

Perception of health care provider relationships was the most intense topic discussed in the interviews. Participants saw various disciplines for different HT related symptoms, which created barriers to building personalized meaningful relationships with providers. Time to see specialists (particularly endocrinologists) was a constraint, as there were often long waits for appointments with insufficient outcomes for symptoms outside of hypothyroidism. Many symptoms of HT were often dismissed by providers and left participants feeling alone and frustrated. This also took a toll on family relationships and decreased the participant's perceived quality of life. When a participant was satisfied with their HT provider, overwhelming positive emotions were expressed furthering the need for intimate patient-provider relationships.

The patient-provider relationship is imperative for treatment satisfaction, especially in life-long chronic illness that require physician surveillance. Trials have shown individuals with chronic illness have consistently better health physiologically, behaviorally, and also subjectively when positive physician-patient communication was present [12]. Patient questionnaires during health care appointments measuring health status, preference for active involvement in treatment decision making, and knowledge of disease and satisfaction with care could be considered [12]. Empathy, trust, and shared decision making are core constructs to support a physician-patient working alliance [13].

Treatment options are limited for HT patients in conventional medicine. Participants were all offered the same synthetic hormone replacement medication and only when the patient requested an alternative (desiccated thyroid) was it discussed. Every patient was euthyroid in this study, yet additional treatment for HT related symptoms was still desired by nearly all. Self-treatment was highly prevalent as participants sought out online resources to assist with their symptoms. Complementary methods such as diet modification and acupuncture were widely accepted and even more treatment options were desired. Social support through online social media groups specific for HT were also commonly used for resources, guidance, and as a comparative tool for treatment plans amongst members.

Health care providers who diagnose and treat HT could benefit from understanding the patient perspective of living with and being treated for this autoimmune disorder. This could strengthen the providerpatient relationship, increase patient-centered care, improve quality of care, and support treatment interventions outlined in the MDM model. Allocating resources that are reputable to patients could ward off the high level of internet searching being done to self-educate on the manifestations and sequelae of HT. Further studies are required to determine if views found in this study are similar in the general HT population.

\section{Disclosure}

The authors declare no conflict of interest.

\section{Acknowledgements}

Support was provided by the University of Florida's Claude D. Pepper Older Americans Independence Center (NIH/NIA P30AG028740). Stephen Anton was previously supported by the Thomas H. Maren Foundation.

\section{References}

1. Caturegli P, De Remigis A, Chuang K, Dembele M, Iwama A, et al. (2013) Hashimoto's thyroiditis: celebrating the centennial through the lens of the Johns Hopkins hospital surgical pathology records. Thyroid 23: 142-150.

2. Garber JR, Cobin RH, Gharib H, Hennessey JV, Klein I, Mechanick, et al. (2012) Clinical practice guidelines for hypothyroidism in adults: cosponsored by the American Association of ClinicalEndocrinologists and the American Thyroid Association. Endocr Pract 18: 988-1028.

3. Müssig K, Künle A, Säuberlich AL, Weinert C, Ethofer T, et al. (2012) Thyroid peroxidase antibody positivity is associated with symptomatic distress in patients with Hashimoto's thyroiditis. Brain Behav Immun 26: 559-563.

4. Promberger R, Hermann M, Ott J (2012) Hashimoto's thyroiditis in patients with normal thyroid-stimulating hormone levels. Expert Review of Endocrinology \& Metabolism 7: 175-179.

5. Cabioglu MT, Cetin BE (2008) Acupuncture and immunomodulation. Am J Chin Med 36: 25-36. [Crossref]

6. Stojanovich L, Marisavljevich D (2008) Stress as a trigger of autoimmune disease. Autoimmun Rev 7: 209-213. [Crossref]

7. Gärtner R, Gasnier BC, Dietrich JW, Krebs B, Angstwurm MW (2002) Selenium supplementation in patients with autoimmune thyroiditis decreases thyroid peroxidase antibodies concentrations. J Clin Endocrinol Metab 87: 1687-1691. 
8. Leppin AL, Montori VM, Gionfriddo MR (2015) Minimally disruptive medicine: a pragmatically comprehensive model for delivering care to patients with multiple chronic conditions. Healthcare (Basel) 3: 50-63.

9. May C, Montori VM, Mair FS (2009) We need minimally disruptive medicine. BMJ 339: b2803. [Crossref]

10. Glaser BG, Strauss AL, Strutzel E (1968) The discovery of grounded theory; strategies for qualitative research. Nursing research 17: 364 .
11. Patton MQ (1990) Qualitative evaluation and research methods. SAGE Publications, inc.

12. Kaplan SH, Greenfield S, Ware JE Jr (1989) Assessing the effects of physician-patient interactions on the outcomes of chronic disease. Med Care 27: S110-127. [Crossref]

13. Fuertes JN, Toporovsky A, Reyes M, Osborne JB (2017) The physician-patient working alliance: Theory, research, and future possibilities. PEC 100: 610-615.

Copyright: $@ 2018$ Lee SA. This is an open-access article distributed under the terms of the Creative Commons Attribution License, which permits unrestricted use, distribution, and reproduction in any medium, provided the original author and source are credited. 